\title{
A Multinational Real-World Study on the Clinical Characteristics of Patients with Type 2 Diabetes Initiating Dapagliflozin in Southern Europe
}

\author{
Gian Paolo Fadini (D) - Nikolaos Tentolouris · Irene Caballero Mateos • \\ Virginia Bellido Castañeda · Cristóbal Morales Portillo
}

Received: November 10, 2019 / Published online: December 20, 2019

(C) The Author(s) 2019

\begin{abstract}
Introduction: A real-world study was performed to describe the clinical characteristics of patients who received dapagliflozin to better understand differences when initiating dapagliflozin in various countries and different prescribing settings.

Methods: We assessed pooled data from observational studies carried out in Italy $(n=2484)$, Spain $(n=564)$ and Greece $(n=87)$. The primary objective was to compare the clinical profile of patients initiating dapagliflozin in the three countries. We also evaluated the percentage of patients who received dapagliflozin in
\end{abstract}

Enhanced Digital Features To view enhanced digital features for this article go to https://doi.org/10.6084/ m9.figshare.11316458.

Electronic supplementary material The online version of this article (https://doi.org/10.1007/s13300019-00744-6) contains supplementary material, which is available to authorized users.

G. P. Fadini $(\bowtie)$

For the DARWIN-T2D Network of the Italian Diabetes Society, Department of Medicine, University of Padova, 35128 Padua, Italy e-mail: gianpaolo.fadini@unipd.it

\section{N. Tentolouris}

1st Department of Propaedeutic Internal Medicine, Medical School, Laiko General Hospital, National and Kapodistrian University of Athens, Athens, Greece clinical practice who satisfied DECLARETIMI 58 enrolment criteria.

Results: In Italy and Spain, around 90\% of patients were receiving metformin vs. $66 \%$ in Greece $(p<0.0001)$. Patients in Greece had lower levels of estimated glomerular filtration rate and lower prevalence rates of retinopathy, prior stroke, acute myocardial infarction, peripheral arterial disease and atherosclerotic cardiovascular disease. Grouping the cohorts by prescribing setting (primary vs. specialist care), baseline $\mathrm{HbA}_{1 \mathrm{c}}$ was lower in primary care $(8.4 \pm 1.7$ vs. $8.7 \pm 1.5$, respectively; $p<0.0001)$. Significantly more patients were receiving other medications for concomitant conditions in specialist care. A total of 1416 patients (48\%) did not meet DECLARE inclusion criteria, while 1561 (52\%) patients met the criteria (Greece $41.05 \%$, Italy $53.19 \%$, Spain $51.35 \%)$.

Conclusions: Significant differences were seen among patients initiating dapagliflozin in southern Europe. Our results suggest that dapagliflozin was being initiated at different

\author{
I. Caballero Mateos \\ Department of Clinical Trials, Hospital Virgen \\ Macarena, Seville, Spain \\ V. Bellido Castañeda \\ Department of Endocrinology and Nutrition, Cruces \\ University Hospital, Bizkaia, Spain \\ C. Morales Portillo \\ Department of Endocrinology and Nutrition, Virgen \\ Macarena University Hospital, Seville, Spain
}


stages of the disease according to the country and prescribing settings. Such geographic heterogeneity may have an impact upon effectiveness of dapagliflozin on glucose lowering, as well as cardiovascular and renal outcomes.

Keywords: Dapagliflozin; Diabetes; Greece; Italy; Real-world; Spain; Therapy

\section{Key Summary Points}

Why carry out this study?

Detailed geographical variations in the clinical characteristics of patients who receive sodium/glucose cotransporter 2 (SGLT2) inhibitors in routine clinical practice are poorly known.

We herein compared phenotypes of patients initiated on dapagliflozin in southern Europe according to country and prescribing patterns.

\section{What was learnt from this study?}

Results suggest that dapagliflozin was being initiated at different stages of the disease according to the country and prescribing settings.

Geographic heterogeneity may impact upon effectiveness of dapagliflozin on glucose lowering, as well as cardiovascular and renal outcomes.

\section{INTRODUCTION}

Type 2 diabetes (T2D) is currently estimated to affect more than 415 million adults globally and, with its increasing prevalence, more than 640 million adults are anticipated to have diabetes by 2040 [1]. As a result of hyperglycaemia and other metabolic abnormalities, T2D is associated with various comorbidities that reduce the quality of life, increase disease-related mortality and complicate its management. Indeed, patients with diabetes are at high risk for adverse outcomes due to cardiovascular disease, including heart failure, and chronic kidney disease [2-5]. Accordingly, minimizing the risk of adverse cardiovascular and renal outcomes has become a new cornerstone in overall management strategies for T2D [6].

Dapagliflozin is a selective sodium/glucose cotransporter 2 inhibitor (SGLT2i) that promotes glucosuria by blocking resorption of glucose in the proximal tubule of the kidney $[7,8]$. Thanks to the resulting reduction in plasma glucose, SGLT2i, including dapagliflozin, are indicated for the management of T2D [9]. In addition to lowering glycated haemoglobin $\left(\mathrm{HbA}_{1 \mathrm{c}}\right)$, SGLT2i also reduce blood pressure and promote weight loss. Four dedicated cardiovascular outcome trials (CVOTs) have shown favourable cardiovascular effects of SGLT2i, mainly in patients with T2D and established cardiovascular disease, including a reduction in the risk of hospitalization for heart failure. Furthermore, SGLT2i may also delay the progression of renal disease [10-13].

The largest CVOT conducted with SGLT2i was DECLARE-TIMI 58 (Dapagliflozin Effect on Cardiovascular Events-Thrombolysis in Myocardial Infarction), which evaluated the effects of dapagliflozin on both cardiovascular and renal outcomes in more than 17,000 patients with (ca. $40 \%$ ) or at risk for (ca. 60\%) atherosclerotic cardiovascular disease (ASCVD) [14]. In this broad population of patients with $\mathrm{T} 2 \mathrm{D}$, the trial showed that dapagliflozin was noninferior to placebo considering the primary safety outcome of major adverse cardiac events (MACE), but it did lead to a significantly lower rate of cardiovascular death or hospitalization for heart failure, with additional findings suggestive of a lower rate of adverse renal events. Results of this and other CVOTs with SGLT2i have now transformed the management of T2D, which is reflected in updated guidelines for T2D. Indeed, SGLT2i have demonstrated CV benefits by reducing the risk of $\mathrm{CV}$ events and mortality in individuals with T2D and established CVD or high CV risk [15]. According to the 2018 American Diabetes Association (ADA)-European Association for the Study of Diabetes (EASD) consensus report, the choice of a second agent to be added to metformin 
should now be driven by the presence or absence of ACVD, heart failure or renal disease, which are all conditions that should favour the use of either an SGLT2i or a glucagon-like peptide 1 (GLP-1) receptor agonist [6]. Thus, there is now a clinical shift from a primary objective of glucose control without hypoglycaemia and weight gain to a new goal of cardiovascular and renal protection [16].

Although randomized clinical trials provide high-quality evidence, their transferability to real-life scenarios is somewhat limited [17]. Real-world studies are also regaining interest owing to their ability to collect large representative data sets on routine clinical settings in a relatively short time. Several real-world observational studies have been carried out with SGLT2 inhibitors. These include CVD-REAL [18], THIN [19] and a Swedish registry [20], as well as a recent real-world study on dapagliflozin with a population similar to that of DECLARE-TIMI 58 [21]. In general, the results of real-world studies with dapagliflozin and other SGLT2i affirm that these agents are associated with more favourable $\mathrm{CV}$ outcomes than other glucose-lowering agents, with lower event rates of hospitalization for heart failure and $\mathrm{CV}$ mortality [22].

The DARWIN-T2D (DApagliflozin Real World evIdeNce in Type 2 Diabetes) study was a multicentre retrospective study in Italy to evaluate the baseline characteristics of patients receiving dapagliflozin vs. comparators, as well as effectiveness in routine clinical practice [23]. The study comprised 17,285 patients initiating dapagliflozin or comparator glucose-lowering medications, and showed that those initiating dapagliflozin were younger, with longer disease duration and higher $\mathrm{HbA}_{1 \mathrm{c}}$, suggesting that dapagliflozin was initially channelled to difficult-to-treat patients. In line with other trials, dapagliflozin reduced $\mathrm{HbA}_{1 \mathrm{c}}$ by $0.7 \%$, body weight by $2.7 \mathrm{~kg}$, systolic blood pressure by $3.0 \mathrm{mmHg}$ and improved albuminuria [24].

To extend these studies to a multinational cohort, we present the results of a real-world study to evaluate the clinical characteristics of patients who received dapagliflozin in different real-world European settings. For this purpose, we assessed pooled data from observational studies carried out in Italy, Spain and Greece. This will thus allow a better understanding of differences in the practice of initiating dapagliflozin in various countries and according to different prescribing settings.

\section{METHODS}

A cohort of 3135 patients with T2D initiating dapagliflozin in Italy $(n=2484)$, Spain $(n=564)$ and Greece $(n=87)$ was analyzed. Recruitment criteria for each country are described below.

\section{Patient Recruitment in Italy}

For Italy, data from the DARWIN-T2D was used. DARWIN-T2D is a multicentre retrospective nationwide investigation involving 46 specialist outpatient clinics in Italy and was promoted by the Italian Diabetes Society. The design of the study has been previously described [24]. Briefly, the primary objective of the study was to describe the baseline characteristics of patients who initiated dapagliflozin following its marketing authorization in Italy (March 2015) until to end of 2016. Inclusion criteria included age 18-80 years, diagnosis of T2D at least 1 year earlier and initiating dapagliflozin $10 \mathrm{mg}$ as add-on to metformin and/or insulin. In Italy, only diabetes specialists were allowed to prescribe dapagliflozin and reimbursement was limited to the combination with insulin and/or metformin.

\section{Patient Recruitment in Spain}

In Spain, dapagliflozin could be prescribed by primary care physicians and no major reimbursement limitations applied. The DAPA-RWE Spain study was conducted at 22 sites in accordance with the ICH Good Clinical Practice guidelines and following approval of the respective ethics committees. Eligible patients were 18-75 years with T2D under stable therapy with glucose-lowering agents. Treatment with dapagliflozin could have started 6 months before inclusion. To be included, the following patient information had to be available at 
baseline: gender, age, date of diagnosis of T2D, weight, height, systolic and diastolic blood pressure, body mass index, concomitant medication, fasting blood glucose, $\mathrm{HbA}_{1 \mathrm{c}}$ and estimated glomerular filtration rate (eGFR). Patients with type 1 diabetes or with gestational diabetes were excluded.

\section{Patient Recruitment in Greece}

Data was used from two studies conducted in Greece, DETECT (DETECTing Treatment Reality of Type 2 Diabetes in Real World Settings) and HEDGE (A national, prospective, non-interventional study of newly diagnosed patients with type 2 diabetes, wHo are starting first line thErapy in the primary care setting, to assess treatment patterns, algorithms and preferences, in GrEece). Data for the group of patients using or starting treatment with SGLT2 inhibitors was extracted. Patients with T2D in DETECT and HEDGE studies were managed in routine care settings across Greece and the studies were conducted at 19 and 38 sites, respectively. As in Spain, in Greece, SGLT2 inhibitors could be prescribed by primary care physicians and there were no major reimbursement limitations. The study population of DETECT comprised patients with T2D who at the time of enrolment were initiating a second-line glucose-lowering therapy after failing their first-line monotherapy (excluding insulin). Inclusion criteria included a signed informed consent form, age at least 18 years and diagnosis of T2D. Exclusion criteria included type 1 diabetes and pregnancy. Patients who were initiating dual therapy after having previously received two different lines of monotherapy or who received a fixed dose combination as first-line treatment were also excluded. In HEDGE study patients with recent (within 12 months) diagnosis of T2D with or without current treatment were included. Further eligibility criteria were a signed informed consent form and age at least 18 years. Exclusion criteria included any other type of diabetes and pregnancy or lactation.

\section{Study Objectives}

Common definitions of the various clinical variables were harmonized among studies. Specifically, chronic kidney disease (CKD) was defined as eGFR (CKD-EPI) of at most $60 \mathrm{ml} /$ $\mathrm{min} / 1.73 \mathrm{~m}^{2}$; CVD was defined as prior history of myocardial infarction, angina, stroke, transient ischemic attack, peripheral arterial disease or revascularization of any arterial site.

We then pooled data from the four countryspecific studies to obtain a unique database of patient characteristics at the time dapagliflozin was prescribed for the first time. The primary objective was to compare the clinical profile of patients initiating dapagliflozin in the three countries. In addition, we compared patients enrolled in Italy, where only diabetes specialists were allowed to prescribe dapagliflozin and reimbursement limitations applied, to patients enrolled in Spain and Greece, where dapagliflozin could be prescribed by primary care physicians and no reimbursement limitations applied. Finally, we wished to evaluate to what extent, for each country, the percentage of patients who received dapagliflozin in clinical practice who satisfied the DECLARE-TIMI 58 enrolment criteria.

\section{Comparison with DECLARE Population}

For this analysis, we used the total population of patients from the three countries, which was divided according to whether relevant DECLARE-TIMI 58 inclusion criteria were met. Inclusion criteria included at least 40 years of age, $\mathrm{HbA}_{1 \mathrm{c}}$ of $6.5-12.0 \%$, creatinine clearance at least $60 \mathrm{ml} / \mathrm{min}$, established ASCVD or two risk factors other than T2D for CVD (age at least 55 years in men and at least 60 years in women, dyslipidaemia, hypertension or current tobacco use).

\section{Statistical Analysis}

Categorical variables were described as count and percentages and compared between countries with chi-square test followed by $2 \times 2$ post hoc comparisons. Quantitative variables were 
described as mean and standard deviation (SD) if normally distributed upon a Shapiro-Wilk test or as median and interquartile range (IQR). Comparisons between countries were made with ANOVA or Kruskal-Wallis test followed by $2 \times 2$ post hoc comparisons. Comparisons between cohorts divided by prescribing setting (primary care versus specialist care) or between study population meeting or not DECLARE inclusion criteria were made with $t$ test or Mann-Whitney test. Hochberg multiple test correction was applied in all analyses. Standardized mean difference (the ratio of difference of means (DECLARE-our sample)/pooled variance) is presented with $95 \%$ confidence intervals as a forest plot. Statistical significance was accepted at $p<0.05$.

\section{Compliance with Ethics Guidelines}

All procedures performed in studies involving human participants were in accordance with the ethical standards of the 1964 Helsinki Declaration and its later amendments or comparable ethical standards. Informed consent was obtained from all individual participants included in the study. For Greece, protocols for the DETECT and the HEDGE studies were approved by all local ethical committees.

For Italy, the protocol of the DARWIN-T2D study was approved by all local ethical committees. For Spain, the protocol of the DAPARWE study was approved by all local ethical committees.

\section{RESULTS}

\section{Clinical Characteristics}

For ease of reference, main baseline clinical characteristics of patients initiating dapagliflozin by country are shown in Fig. 1, and more detailed data are shown in Table 1. A significant difference among countries was found for age, with patients being slightly older in Spain vs. Italy and Greece. Duration of diabetes was lowest in Greece (at 4 years) and highest in Spain (at 14 years). Baseline $\mathrm{HbA}_{1 \mathrm{c}}$ and fasting

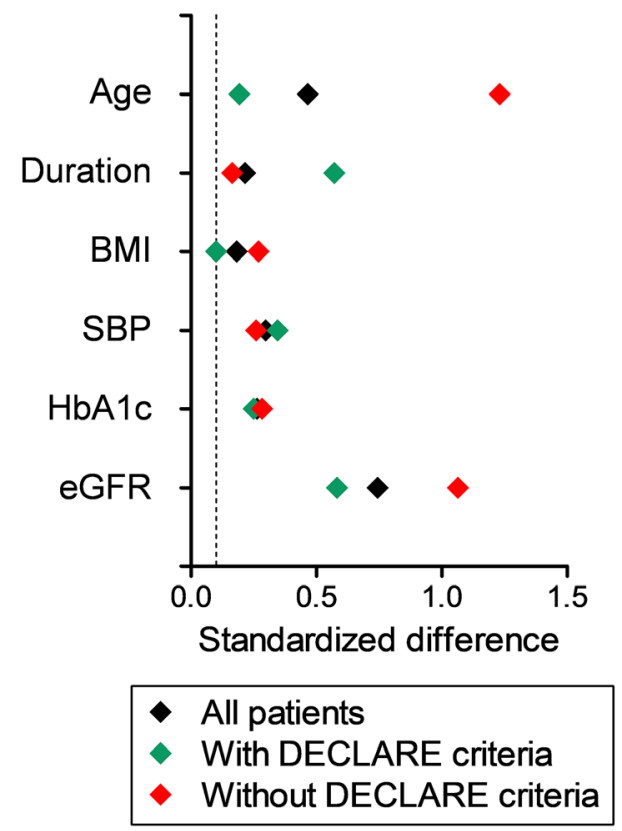

Fig. 1 Forest plot of standardized mean differences of the DECLARE-TIMI 58 population vs. the entire population and those meeting or not meeting inclusion criteria

plasma glucose (FPG) were both lower in Greece than in Italy and Spain. Body mass index (BMI) was significantly higher in Spain. Patients in Greece had significantly lower blood pressure compared to Italy and Spain. Patients in Greece also had the highest levels of low-density lipoprotein (LDL)-cholesterol.

\section{Concomitant Medications}

Significant differences among countries were found for concomitant glucose-lowering medications (Table 1). In Italy and Spain, around $90 \%$ of patients were receiving metformin vs. $66 \%$ in Greece $(p<0.0001)$. No patients in Greece were receiving insulin, compared to $42 \%$ in Spain and $54 \%$ in Italy. No patients in Italy were concomitantly receiving a sulfonylurea or GLP-1RA, whereas, in Spain, 28\% of patients were receiving a sulfonylurea and $17 \%$ a GLP1RA. Regarding drugs for control of concomitant risk factors, patients in Italy received more medications overall than those in the other two countries. 
Table 1 Detailed baseline patient characteristics, concomitant therapies and concomitant medications by country

\begin{tabular}{|c|c|c|c|c|c|c|}
\hline Variable & & Total & Greece $(N=87)$ & Italy $(N=2484)$ & Spain $(N=564)$ & $P$ value \\
\hline Duration & Mean (SD) & $12.4(8.05)$ & $4.1(4.3)^{* \dagger}$ & $12.3(8.0)^{\#}$ & $14.0(7.7)$ & $<0.0001$ \\
\hline Male sex & $N(\%)$ & $1840(58.0)$ & $55(57.9)$ & $1449(58.3)$ & $336(56.6)$ & 0.735 \\
\hline Age & Mean (SD) & $60.6(9.5)$ & $58.8(9.9)^{\dagger}$ & $60.4(9.4)^{\#}$ & $61.8(10.0)$ & 0.0009 \\
\hline Weight & Mean (SD) & $91.8(18.3)$ & $93.1(18.4)$ & $91.7(18.5)$ & $92.0(17.5)$ & 0.726 \\
\hline Height & Mean (SD) & $166.6(9.8)$ & $168.6(10.6)^{\dagger}$ & $166.9(9.8)^{\#}$ & $164.9(9.4)$ & $<0.0001$ \\
\hline BMI & Mean (SD) & $33.1(5.9)$ & $32.8(6.1)$ & $32.9(5.9)^{\#}$ & $33.8(5.8)$ & 0.004 \\
\hline Waist & Mean (SD) & $110.7(13.5)$ & $108.8(13.8)$ & $111.2(13.3)$ & $108.0(14.7)$ & $0.036^{\S}$ \\
\hline SBP & Mean $(\mathrm{SD})$ & $139.9(18.8)$ & $133.0(14.3)^{* \dagger}$ & $140.1(19.3)$ & $140.3(18.0)$ & 0.001 \\
\hline DBP & Mean (SD) & $80.4(10.6)$ & $80.0(9.9)$ & $80.4(10.4)$ & $80.5(11.2)$ & 0.924 \\
\hline HR & Mean (SD) & $79.9(12.4)$ & $75.9(9.7)^{* \dagger}$ & $80.1(12.8)$ & $80.8(12.5)$ & 0.006 \\
\hline FPG & Mean (SD) & $176.6(58.1)$ & $156.3(56.2)^{* \dagger}$ & $178.3(56.5)$ & $173.9(62.8)$ & 0.001 \\
\hline $\mathrm{HbA}_{\mathrm{lc}}$ & Mean (SD) & $8.6(1.5)$ & $7.4(1.5)^{* \dagger}$ & $8.7(1.5)$ & $8.5(1.6)$ & $<0.0001$ \\
\hline TC & Mean (SD) & $176.8(40.6)$ & $185.8(37.3)$ & $176.7(40.1)$ & $175.7(42.3)$ & 0.134 \\
\hline $\mathrm{HDL}$ & Mean (SD) & $45.1(13.1)$ & $45.3(13.6)$ & $45.4(12.5)$ & $44.3(14.5)$ & 0.265 \\
\hline TG & Mean (SD) & $178.7(139.6)$ & $158.3(80.7)$ & $176.3(138.0)$ & $188.0(149.9)$ & 0.108 \\
\hline LDLC & Mean (SD) & $96.6(33.6)$ & $108.6(31.8)^{* \dagger}$ & $96.4(33.5)$ & $95.8(33.9)$ & 0.014 \\
\hline SGOT & Mean (SD) & $25.5(16.3)$ & $26.4(19.5)$ & $25.06(17.4)$ & $26.2(13.2)$ & 0.439 \\
\hline SGPT & Mean (SD) & $32.7(28.3)$ & $32.1(30.4)$ & $33.23(32.5)$ & $31.8(18.5)$ & 0.636 \\
\hline Metformin & $N(\%)$ & $2836(89.4)$ & $63(66.3)^{* \dagger}$ & $2262(91.1)^{\#}$ & $511(86.0)$ & $<0.0001$ \\
\hline Insulin & $N(\%)$ & $1605(50.6)$ & $0(0.0)^{* \dagger}$ & $1353(54.5)^{\#}$ & $252(42.4)$ & $<0.0001$ \\
\hline SU & $N(\%)$ & $167(5.3)$ & $2(2.1)^{* \dagger}$ & $0(0.0)^{\#}$ & $165(27.8)$ & $<0.0001$ \\
\hline DPP4i & $N(\%)$ & $3(0.1)$ & $3(3.2)^{* \dagger}$ & $0(0.0)^{\#}$ & $0(0.0)$ & $<0.0001$ \\
\hline GLP-1RA & $N(\%)$ & $100(3.2)$ & $0(0) \mathrm{a}$ & $0(0.0)^{\#}$ & $100(16.8)$ & $<0.0001$ \\
\hline Pioglitazone & $N(\%)$ & $23(0.7)$ & $0(0)$ & $0(0.0)$ & $23(3.9)$ & $<0.0001$ \\
\hline Acarbose & $N(\%)$ & $1(0.0)$ & $0(0.0)$ & $0(0.0)$ & $1(0.2)$ & 0.114 \\
\hline $\mathrm{ACEi}-\mathrm{ARBs}$ & $N(\%)$ & $1771(66.5)$ & $44(46.3)^{* \dagger}$ & $1369(69.4)^{\#}$ & $358(60.3)$ & $<0.0001$ \\
\hline Beta-blockers & $N(\%)$ & $706(26.5)$ & $26(27.4)^{\dagger}$ & $584(29.6)^{\#}$ & $96(16.2)$ & $<0.0001$ \\
\hline $\mathrm{CCB}$ & $N(\%)$ & $499(18.7)$ & $14(14.7)^{\dagger}$ & $445(22.5)^{\#}$ & $40(6.73)$ & $<0.0001$ \\
\hline Statin-fibrates & $N(\%)$ & $1683(63.2)$ & $56(59.0)^{\dagger}$ & $1366(69.2)^{\#}$ & $261(43.9)$ & $<0.0001$ \\
\hline Anti-platelet & $N(\%)$ & $1045(39.2)$ & $14(14.7)^{*}$ & $880(44.6)^{\#}$ & $151(25.4)$ & $<0.0001$ \\
\hline Creatinine & Mean (SD) & $1.0(4.9)$ & $0.9(0.2)$ & $0.9(0.2)$ & $1.3(9.1)$ & 0.133 \\
\hline eGFR & Mean (SD) & $99.8(28.6)$ & $66.0(4.4)$ & $107.0(27.0)^{\#}$ & $82.7(24.8)$ & $<0.0001$ \\
\hline Urinary albumin & Median (IQR) & $14.0(6.5)$ & $5.0(5.5)$ & $15.8(7.1-61.1)^{\#}$ & $12(4.0-33.0)$ & $<0.0001$ \\
\hline
\end{tabular}


Table 1 continued

\begin{tabular}{llccccc}
\hline Variable & & Total & Greece $(\boldsymbol{N}=\mathbf{8 7})$ & Italy $(\boldsymbol{N}=\mathbf{2 4 8 4})$ & Spain $(\boldsymbol{N}=\mathbf{5 6 4})$ & $\boldsymbol{P}$ value \\
\hline Retinopathy & $N(\%)$ & $509(20.5)$ & $1(2.6)^{*}$ & $430(22.0)^{\#}$ & $78(15.8)$ & 0.000 \\
Stroke & $N(\%)$ & $71(2.2)$ & $0(0.0)$ & $41(1.7)^{\#}$ & $30(5.1)$ & $<0.0001$ \\
AMI & $N(\%)$ & $285(10.7)$ & $7(7.4)$ & $192(9.7)^{\#}$ & $86(14.5)$ & 0.002 \\
HF & $N(\%)$ & $84(3.2)$ & $1(1.1)$ & $61(3.1)$ & $22(3.7)$ & 0.370 \\
PAD & $N(\%)$ & $176(10.3)$ & $2(2.1)^{*}$ & $137(13.5)^{\#}$ & $37(6.2)$ & $<0.0001$ \\
ASCVD & $N(\%)$ & $361(21.7)$ & $9(9.5)^{* \dagger}$ & $223(22.9)$ & $129(21.8)$ & 0.010 \\
\hline
\end{tabular}

$B M I$ body mass index, $S B P$ systolic blood pressure, $D B P$ diastolic blood pressure, $H R$ heart rate, $F P G$ fasting plasma glucose, $H b A_{1 c}$ glycated haemoglobin, $T C$ total cholesterol, $H D L$ high-density lipoprotein, $T G$ triglycerides, $L D L C$ low-density lipoprotein-cholesterol, $S G O T$ serum glutamic oxaloacetic transaminase, $S G P T$ serum glutamic pyruvic transaminase, $S U$ sulfonylurea, DPP4i dipeptidyl peptidase 4 inhibitor, GLP-1RA glucagon-like peptide 1 receptor agonist, $A C E i-A R B s$ angiotensin-converting enzyme inhibitor and an angiotensin receptor blocker, $C C B$ calcium channel blockers, $e G F R$ estimated glomerular filtration rate, $A M I$ acute myocardial infarction, $H F$ heart failure, $P A D$ peripheral arterial disease, $A S C V D$ atherosclerotic cardiovascular disease

$\S$ Not significant after Hochberg multiple test correction

${ }^{*} p<0.05$ post hoc comparison Greece vs. Italy

${ }^{\dagger} p<0.05$ post hoc comparison Greece vs. Spain

\# $p<0.05$ post hoc comparison Italy vs. Spain

\section{Comorbidities}

Significant differences among countries were found in comorbidities (Table 1). Patients in Greece had lower levels of eGFR and lower prevalence rates of retinopathy, prior stroke, acute myocardial infarction, peripheral arterial disease and ASCVD. More than one in five patients in Italy had diagnosed retinopathy, compared to $16 \%$ in Spain and 2.6\% in Greece. Cardiac comorbidities had roughly similar proportions in Italy and Spain.

\section{Clinical Features Based on Prescribing Setting}

Grouping the cohorts by prescribing setting (specialist vs. primary care) led to some differences being seen in baseline clinical characteristics (Table 2). In particular, lower age and BMI were seen in specialist care compared to primary care. Baseline $\mathrm{HbA}_{1 \mathrm{c}}$ was also lower in primary vs. specialist $(8.4 \pm 1.6$ vs. $8.7 \pm 1.5$, respectively; $p<0.0001$ ), with higher FPG in the latter setting. No significant differences were seen for the other laboratory variables. Differences in the frequency of concomitant medications were seen for all medications, which may reflect local reimbursement limitations more than the healthcare setting (Table 2). In fact, more patients in specialist care were on the glucoselowering therapies metformin and insulin, and none on the remaining glucose-lowering therapies, compared to the two countries where these medications were prescribed by primary care physicians. Significantly more patients in specialist care were also on other medications for concomitant conditions compared to those in primary care. Lastly, differences in the proportion of patients with comorbidities were also seen. Of note, eGFR and urinary albumin levels were lower in primary care, and fewer patients in these countries also had concomitant retinopathy and peripheral artery disease. 
Table 2 Baseline patient characteristics, concomitant therapies and concomitant medications by prescribing setting

\begin{tabular}{|c|c|c|c|c|c|}
\hline Variable & & Total & Specialist care $(N=2484)$ & Primary care $(N=689)$ & $P$ value* \\
\hline Duration & Mean (SD) & $12.4(8.1)$ & $12.2(8.0)$ & $12.6(8.1)$ & 0.322 \\
\hline Male sex & $N(\%)$ & $1840(58.0)$ & $1449(58.3)$ & $391(56.8)$ & 0.015 \\
\hline Age & Mean (SD) & $60.6(9.5)$ & $60.4(9.4)$ & $61.4(10.0)$ & 0.544 \\
\hline Weight & Mean (SD) & $91.8(18.3)$ & $91.7(18.5)$ & $92.2(17.6)$ & 0.000 \\
\hline Height & Mean (SD) & $166.6(9.8)$ & $166.9(9.8)$ & $165.4(9.6)$ & 0.004 \\
\hline BMI & Mean (SD) & $33.1(5.9)$ & $32.9(5.9)$ & $33.7(5.8)$ & 0.011 \\
\hline Waist & Mean (SD) & $110.7(13.5)$ & $111.2(13.3)$ & $108.4(14.2)$ & 0.275 \\
\hline SBP & Mean (SD) & $139.9(18.8)$ & $140.1(19.3)$ & $139.2(17.7)$ & 0.994 \\
\hline DBP & Mean $(S D)$ & $80.4(10.6)$ & $80.4(10.4)$ & $80.4(11.0)$ & 0.771 \\
\hline $\mathrm{HR}$ & Mean (SD) & $79.9(12.4)$ & $80.1(12.8)$ & $79.8(12.1)$ & 0.009 \\
\hline FPG & Mean (SD) & $176.6(58.1)$ & $178.3(56.5)$ & $171.6(62.2)$ & $<0.0001$ \\
\hline $\mathrm{HbA}_{1 \mathrm{c}}$ & Mean (SD) & $8.6(1.5)$ & $8.7(1.5)$ & $8.4(1.6)$ & 0.934 \\
\hline TC & Mean (SD) & $176.8(40.6)$ & $176.7(40.1)$ & $176.9(41.9)$ & 0.128 \\
\hline HDL & Mean (SD) & $45.1(13.1)$ & $45.4(12.5)$ & $44.4(14.4)$ & 0.214 \\
\hline TG & Mean (SD) & $178.7(139.6)$ & $176.3(138.0)$ & $184.5(143.4)$ & 0.584 \\
\hline LDLC & Mean (SD) & $96.6(33.6)$ & $96.4(33.5)$ & $97.3(33.9)$ & 0.201 \\
\hline SGOT & Mean (SD) & $25.5(16.2)$ & $25.1(17.4)$ & $26.2(13.8)$ & 0.342 \\
\hline SGPT & Mean (SD) & $32.7(28.3)$ & $33.2(32.5)$ & 31.8 (19.58) & 0.065 \\
\hline Metformin & $N(\%)$ & $2836(89.4)$ & $2262(91.1)$ & $574(83.3)$ & $<0.0001$ \\
\hline Insulin & $N(\%)$ & $1605(50.6)$ & $1353(54.5)$ & $252(36.6)$ & $<0.0001$ \\
\hline SU & $N(\%)$ & $167(5.3)$ & $0(0)$ & $167(24.2)$ & 0.456 \\
\hline DPP4i & $N(\%)$ & $3(0.1)$ & $0(0)$ & $3(0.4)$ & 0.000 \\
\hline GLP-1RA & $N(\%)$ & $100(3.2)$ & $0(0)$ & $100(14.5)$ & $<0.0001$ \\
\hline Pioglitazone & $N(\%)$ & $23(0.7)$ & $0(0)$ & $23(3.3)$ & 0.005 \\
\hline Acarbose & $N(\%)$ & $1(0.0)$ & $0(0)$ & $1(0.2)$ & 0.748 \\
\hline ACEi-ARBs & $N(\%)$ & $1771(66.5)$ & $1369(69.4)$ & $402(58.4)$ & $<0.0001$ \\
\hline Beta-blockers & $N(\%)$ & $706(26.5)$ & $584(29.6)$ & $122(17.7)$ & 0.180 \\
\hline CCB & $N(\%)$ & $499(18.7)$ & $445(22.5)$ & $54(7.8)$ & $<0.0001$ \\
\hline Statin-fibrates & $N(\%)$ & $1683(63.2)$ & $1366(69.2)$ & $317(46.0)$ & $<0.0001$ \\
\hline Anti-platelet & $N(\%)$ & $1045(39.2)$ & $880(44.6)$ & $165(24.0)$ & $<0.0001$ \\
\hline Creatinine & Mean (SD) & $1.0(4.9)$ & $0.9(0.2)$ & $1.3(8.6)$ & 0.001 \\
\hline eGFR & Mean (SD) & $99.8(28.58)$ & $107.0(27.0)$ & $82.7(24.8)$ & $<0.0001$ \\
\hline Urinary albumin & Median & $14.0(6.0-52.2)$ & $15.8(7.1-61.2)$ & $12(4.3)$ & $<0.0001$ \\
\hline
\end{tabular}


Table 2 continued

\begin{tabular}{llcccc}
\hline Variable & & Total & Specialist care $(\boldsymbol{N}=\mathbf{2 4 8 4})$ & Primary care $(\boldsymbol{N}=\mathbf{6 8 9})$ & $\boldsymbol{P}$ value $^{*}$ \\
\hline Retinopathy & $N(\%)$ & $509(20.5)$ & $430(22.0)$ & $79(14.9)$ & 0.058 \\
Stroke & $N(\%)$ & $71(2.2)$ & $41(1.7)$ & $30(4.4)$ & $<0.0001$ \\
AMI & $N(\%)$ & $285(10.7)$ & $192(9.7)$ & $93(13.5)$ & $<0.0001$ \\
HF & $N(\%)$ & $84(3.2)$ & $61(3.1)$ & $23(3.3)$ & $<0.0001$ \\
PAD & $N(\%)$ & $176(10.3)$ & $137(13.5)$ & $39(5.7)$ & $<0.0001$ \\
ASCVD & $N(\%)$ & $361(21.7)$ & $223(22.9)$ & $138(20.1)$ & $<0.0001$ \\
\hline
\end{tabular}

Abbreviations are defined in Table 1

*Post hoc comparison between groups

\section{Comparison with DECLARE-TIMI 58 Population}

Comparison of the DECLARE-TIMI 58 population with patients who received dapagliflozin in routine care is of interest as it can provide information about the generalizability of trial findings. For this analysis, we first used the total population of patients from the three countries, which was divided according to whether the relevant DECLARE-TIMI 58 inclusion criteria were met. A total of 1416 patients (48\%) did not meet DECLARE-TIMI 58 inclusion criteria, while $1561(52 \%)$ patients met the criteria (Greece $41.05 \%$, Italy 53.19\%, Spain 51.35\%). Data for selected variables were then compared between the three subgroups (Table 3). Forest plots of standardized mean differences of the DECLARE-TIMI 58 population vs. the entire population and those meeting or not DECLARETIMI 58 inclusion criteria are shown in Fig. 2. Patients fulfilling DECLARE-TIMI 58 enrolment criteria still showed important significant differences compared to the DECLARE-TIMI 58 population. Significant differences were found for concomitant glucose-lowering agents among patients meeting or not meeting DECLARE-TIMI 58 criteria, and especially use of DPP4i and sulfonylurea as few patients were on either medication in our cohorts, compared to $16.5 \%$ and $42.1 \%$, respectively, in DECLARE-
TIMI 58. More patients in DECLARE-TIMI 58 were also receiving concomitant cardiovascular medications vs. both our subpopulations, which is expected, since patients in DECLARETIMI 58 were enrolled on the basis of cardiovascular risk criteria.

\section{DISCUSSION}

The present analysis was undertaken to evaluate specific characteristics of 3135 patients receiving dapagliflozin in different real-world settings in southern Europe using pooled data from observational studies carried out in Italy, Spain and Greece. Significant differences among countries were found for several baseline characteristics, including age, duration of disease, and glucose control. Substantial differences were also found for use of concomitant glucoselowering medications, which might largely be explained by differences in local reimbursement limitations in the three countries. At the time of the study, in Italy, only diabetes specialists were allowed to prescribe dapagliflozin and reimbursement was limited further to the combination with insulin and/or metformin. In contrast, in both Spain and Greece, dapagliflozin can be prescribed by primary care physicians with major limitations for reimbursement. The differences in baseline characteristics, and especially $\mathrm{HbA}_{1 \mathrm{c}}$, are also of interest as this was 
Table 3 Comparison between baseline characteristics of the pooled study population (meeting or not DECLARE inclusion criteria) with that of DECLARE

\begin{tabular}{|c|c|c|c|c|}
\hline Variable & All patients & $\begin{array}{l}\text { Not satisfying DECLARE } \\
\text { inclusion criteria }(N=1416)\end{array}$ & $\begin{array}{l}\text { Satisfying DECLARE } \\
\text { inclusion criteria }(N=1561)\end{array}$ & $\begin{array}{l}\text { DECLARE } \\
\text { population } \\
(\boldsymbol{N}=\mathbf{8 5 8 2})\end{array}$ \\
\hline Male sex, $N(\%)$ & $1721(57.8)$ & $739(52.2)^{*}$ & $982(62.9)$ & $5411(63.1)$ \\
\hline $\mathrm{PAD}, N(\%)$ & $176(10.9)$ & $45(6.3)$ & $131(14.6)^{*}$ & $522(6.1)$ \\
\hline $\begin{array}{l}\text { Metformin, } \\
\quad N(\%)\end{array}$ & 2656 (89.2) & $1274(90.0)^{*}$ & $1382(88.5)^{*}$ & $7020(81.8)$ \\
\hline Insulin, $N(\%)$ & $1504(50.5)$ & $664(46.9)^{*}$ & $840(53.8)^{*}$ & $3567(41.6)$ \\
\hline SU, $N(\%)$ & $167(5.6)$ & $85(6.0)^{*}$ & $82(5.3)^{*}$ & $3615(42.1)$ \\
\hline DPP4i, $N(\%)$ & $3(0.1)$ & $2(0.1)^{*}$ & $1(0.1)^{*}$ & $1418(16.5)$ \\
\hline GLP-1RA (\%) & $100(3.4)$ & $53(3.7)$ & $47(3.01)^{*}$ & $397(4.6)$ \\
\hline $\begin{array}{l}\text { ACEi-ARBs, } \\
\quad N(\%)\end{array}$ & $1771(66.5)$ & $652(56.7)^{*}$ & $1119(73.9)^{*}$ & $6977(81.3)$ \\
\hline $\begin{array}{l}\text { Beta-blockers, } \\
\quad N(\%)\end{array}$ & $706(26.5)$ & $235(20.4)^{*}$ & $471(31.1)^{*}$ & $4498(52.4)$ \\
\hline $\begin{array}{l}\text { Statin-fibrates, } \\
\quad N(\%)\end{array}$ & $1683(63.2)$ & $636(55.4)^{*}$ & $1047(69.2)^{*}$ & $6432(74.9)$ \\
\hline $\begin{array}{l}\text { Anti-platelets, } \\
\qquad N(\%)\end{array}$ & $1045(39.2)$ & $331(28.8)^{*}$ & $714(47.2)^{*}$ & $5245(61.1)$ \\
\hline $\begin{array}{l}\text { Duration, mean } \\
\quad(\mathrm{SD})\end{array}$ & $12.3(8.03)$ & $10.1(7.0)^{*}$ & $14.2(8.4)^{*}$ & $11.0(5.0)$ \\
\hline $\begin{array}{l}\text { Age, mean } \\
\text { (SD) }\end{array}$ & $60.3(9.7)$ & $55.0(9.6)^{*}$ & $65.2(6.7)^{*}$ & $63.9(6.8)$ \\
\hline $\begin{array}{l}\text { BMI, mean } \\
\quad(\mathrm{SD})\end{array}$ & $33.2(5.9)$ & $32.7(5.7)^{*}$ & $32.7(5.7)^{*}$ & $32.1(6.0)$ \\
\hline $\begin{array}{l}\text { SBP, mean } \\
\quad(\mathrm{SD})\end{array}$ & $139.9(18.9)$ & $139.3(20.8)^{*}$ & $140.5(17.0)^{*}$ & $135.1(15.3)$ \\
\hline $\begin{array}{l}\mathrm{HbA}_{1 \mathrm{c}} \text {, mean } \\
\quad(\mathrm{SD})\end{array}$ & $8.6(1.5)$ & $8.7(1.2)^{*}$ & $8.6(1.2)^{*}$ & $8.3(1.2)$ \\
\hline $\begin{array}{l}\text { eGFR, mean } \\
(\mathrm{SD})\end{array}$ & $99.4(28.6)$ & $104.0(27.7)^{*}$ & $95.6(27.7)^{*}$ & $85.4(15.8)$ \\
\hline
\end{tabular}

Abbreviations are defined in Table 1

${ }^{*} P<0.05$

much lower in Greece vs. the other two countries. Altogether, the differences in age, diabetes duration and baseline $\mathrm{HbA}_{1 \mathrm{c}}$ suggest that dapagliflozin was being used at different disease stages in different clinical settings. This is confirmed by the diverse pattern of concomitant 


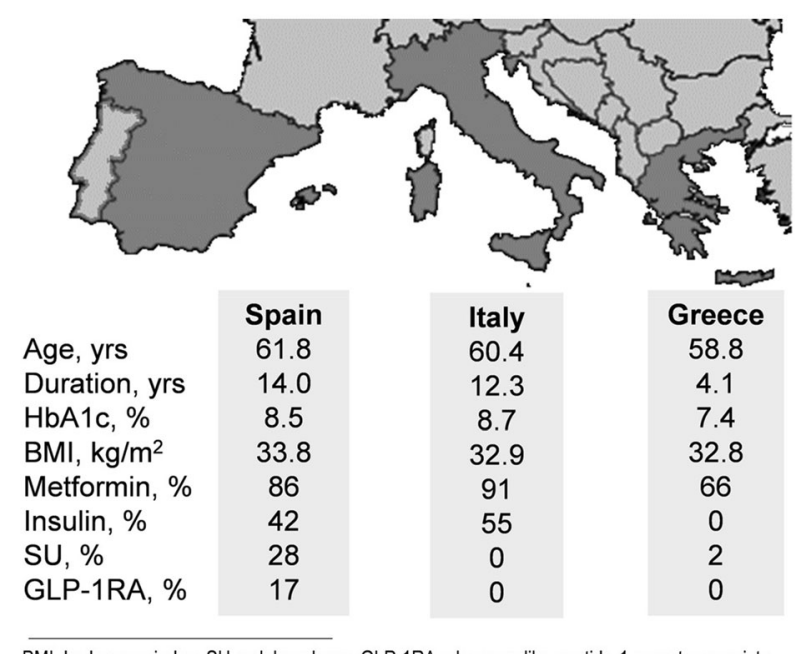

BMI, body mass index. SU, sulphonylurea. GLP-1RA, glucagon-like peptide-1 receptor agonists

Fig. 2 Key baseline patient characteristics and concomitant therapies by country

glucose-lowering medications. Importantly, country-specific reimbursement criteria were likely to be driving differences in choice of combination therapy. Our results also highlight that geographic heterogeneity is present with regards to prescribing practices for dapagliflozin, which may have an impact on its effectiveness in real-world settings outside of controlled clinical trials.

Results of the large DARWIN-T2D study [24], which assessed the clinical profile of patients receiving a new prescription of dapagliflozin or other glucose-lowering medications in routine clinical practice in over 17,000 patients in Italy, suggested that diabetes care specialists had been initially prescribing dapagliflozin to patients considered difficult-to-treat, at least in the study period (2015-2016).

The cardiovascular safety of dapagliflozin was analyzed in the large DECLARE-TIMI 58 trial involving over 17,000 patients followed for a median of 4.2 years, over 10,000 of whom did not have ASCVD. The DECLARE population thus represents a broad group of patients with type 2 diabetes, and who had or were at risk for ASCVD. Our analysis from the pooled patient population from Italy, Spain and Greece indicates that many differences in baseline characteristics were present between the multinational south Europe population and that of DECLARETIMI 58 population, independently of whether or not patients met inclusion criteria for DECLARE-TIMI 58. Interestingly, a high proportion of patients initiating dapagliflozin in routine clinical practice satisfied DECLARETIMI 58 enrolment criteria. For comparison, Wittbrodt et al. reported that the eligibility of patients in the Diabetes Collaborative Registry was much lower for other CVOTs with SGLT2i, thereby suggesting a higher generalizability of the DECLARE-TIMI 58 population in the southern European population [25]. However, it should be noted that, even among patients who satisfied DECLARE-TIMI 58 eligibility criteria and received dapagliflozin, significant differences were found compared to the actual DECLARE-TIMI 58 population. Therefore, transferability of CVOT results to clinical practice needs to be accurately scrutinized. In this regard, the results of retrospective studies such as CVD-REAL [18], THIN [19] and a Swedish registry [20] are reassuring that the benefits observed in SGLT2i CVOTs can be translated to patients receiving dapagliflozin in routine care.

A limitation of this study is that the cohort for Greece is relatively small so it might be less representative of the real-world patient profile in Greece. In addition, differences in inclusion criteria for the considered studies might also be considered a selection bias. 


\section{CONCLUSIONS}

Our pooled multinational real-world study found significant geographical differences among patients initiating dapagliflozin that help one to understand prescribing practices and patterns. The results suggest that dapagliflozin was being initiated at different stages of the disease according to the country-specific and prescribing settings. While country-specific reimbursement criteria may be at the basis of the observed differences in prescription of combination therapies, it also suggests that such geographic heterogeneity may have an impact upon effectiveness of dapagliflozin on glucose lowering, as well as cardiovascular and renal outcomes. To address these issues, we plan to extend the present baseline analysis to assess the effectiveness of dapagliflozin by country and prescribing setting.

\section{ACKNOWLEDGEMENTS}

We thank the participants of the study and all centres involved in the four studies (see supplementary material).

Funding. AstraZeneca sponsored the analysis of data obtained in the four trials: DARWINT2D Sponsored by "Italian Diabetes Society" and partially supported by a grant from AstraZeneca, DAPA-RWE sponsored by "Fundación Pública Andaluza para la Gestión de la Investigación en Salud de Sevilla" and partially supported by a grant from AstraZeneca, DETECT sponsored by AstraZeneca, HEDGE sponsored by AstraZeneca. All authors had full access to all of the data in this study and take complete responsibility for the integrity of the data and accuracy of the data analysis. All authors had full access to all of the data in this study and take complete responsibility for the integrity of the data and accuracy of the data analysis. The journal's Rapid Service Fee was covered by AstraZeneca.

Authorship. All named authors meet the International Committee of Medical Journal
Editors (ICMJE) criteria for authorship for this article, take responsibility for the integrity of the work as a whole, and have given their approval for this version to be published.

Medical Writing and/or Editorial Assistance. Editorial assistance in the preparation of this article was provided by Patrick Moore, PhD on behalf of Edra S.p.A., and unconditionally funded by AstraZeneca.

Disclosures. Gian Paolo Fadini has received research grants or a speaker honorarium from Abbott, AstraZeneca, Boehringer, Lilly, Novo Nordisk, Mundipharma, Novartis, Sanofi. Nikolaos Tentolouris has participated in advisory panels for Merck Sharp Dohme, AstraZeneca, Sanofi, Novo Nordisk, ELPEN, Eli Lilly, Servier, Boehringer Ingelheim and Novartis; and has received research support from Merck Sharp Dohme, Eli Lilly, Novo Nordisk, Sanofi, Pfizer, AstraZeneca, Janssen, Cilag, GlaxoSmithKline and Novartis. Irene Caballero Mateos has nothing to disclose. Virginia Bellido Castañeda has received honoraria for consulting and/or speaking (Sanofi, NovoNordisk, Lilly, AstraZeneca, Janssen, Boehringer, MSD, Abbott and Esteve). Cristóbal Morales Portillo has participated in advisory panels for NovoNordisk, Lilly, MSD, Boehringer, AstraZeneca, Sanofi, Abbot; has received research grants from NovoNordisk, Sanofi, AstraZeneca, Pzifer, Lilly, Merck, Lexicon, FPS, Hanmi, Janssen, Boehringer, Takeda, Roche, Theracos, LeeGanz; speaker honorarium from Sanofi, Novonordisk, AstraZeneca, Roche, Lilly, Boehringher, MSD, Ferrer, Janssen, Abbot.

Compliance with Ethics Guidelines. All procedures performed in studies involving human participants were in accordance with the ethical standards of the 1964 Helsinki Declaration and its later amendments or comparable ethical standards. Informed consent was obtained from all individual participants included in the study. For Greece, protocols for the DETECT and the HEDGE studies were approved by all local ethical committees. For Italy, the protocol of the DARWIN-T2D study was approved by all local ethical committees. For 
Spain, the protocol of the DAPA-RWE study was approved by all local ethical committees.

Data Availability. The data sets generated during and/or analyzed during the current study are available from the corresponding author on reasonable request.

Open Access. This article is distributed under the terms of the Creative Commons Attribution-NonCommercial 4.0 International License (http://creativecommons.org/licenses/ by-nc/4.0/), which permits any noncommercial use, distribution, and reproduction in any medium, provided you give appropriate credit to the original author(s) and the source, provide a link to the Creative Commons license, and indicate if changes were made.

\section{REFERENCES}

1. Ogurtsova K, da Rocha Fernandes JD, Huang Y, et al. IDF diabetes atlas: global estimates for the prevalence of diabetes for 2015 and 2040. Diabetes Res Clin Pract. 2017;128:40-50.

2. Ahmad FS, Ning H, Rich JD, et al. Hypertension, obesity, diabetes, and heart failure-free survival: the cardiovascular disease lifetime risk pooling project. JACC Heart Fail. 2016;4(12):911-9.

3. Donahoe SM, Stewart GC, McCabe CH, et al. Diabetes and mortality following acute coronary syndromes. JAMA. 2007;298(7):765-75.

4. Ritz E, Orth SR. Nephropathy in patients with type 2 diabetes mellitus. $\mathrm{N}$ Engl $\mathrm{J}$ Med. 1999;341(15):1127-33.

5. Roger VL, Go AS, Lloyd-Jones DM, et al. Heart disease and stroke statistics-2011 update: a report from the American Heart Association. Circulation. 2011;123(4):e18-209.

6. Davies MJ, Dalessio DA, Fradkin J, et al. Management of hyperglycemia in type 2 diabetes, 2018. A consensus report by the American Diabetes Association (ADA) and the European Association for the Study of Diabetes (EASD). Diabetes Care. 2018;41(12):2669-701.

7. Han S, Hagan DL, Taylor JR, et al. Dapagliflozin, a selective SGLT2 inhibitor, improves glucose homeostasis in normal and diabetic rats. Diabetes. 2008;57(6):1723-9.
8. Komoroski B, Vachharajani N, Feng Y, et al. Dapagliflozin, a novel, selective SGLT2 inhibitor, improved glycemic control over 2 weeks in patients with type 2 diabetes mellitus. Clin Pharmacol Ther. 2009;85(5):513-9.

9. Dapagliflozin. Summary of product characteristics. https://www.ema.europa.eu/en/documents/productinformation/forxiga-epar-product-information_it.pdf. Accessed 17 Dec 2019.

10. Mahaffey KW, Neal B, Perkovic V, et al. Canagliflozin for primary and secondary prevention of cardiovascular events: results from the CANVAS program (Canagliflozin Cardiovascular Assessment Study). Circulation. 2018;137(4):323-34.

11. Neal B, Perkovic V, Mahaffey KW, et al. Canagliflozin and cardiovascular and renal events in type 2 diabetes. N Engl J Med. 2017;377(7):644-57.

12. Perkovic V, Jardine MJ, Neal B, et al. Canagliflozin and renal outcomes in type 2 diabetes and nephropathy. N Engl J Med. 2019;380(24):2295-306.

13. Zinman B, Lachin JM, Inzucchi SE. Empagliflozin, cardiovascular outcomes, and mortality in type 2 diabetes. N Engl J Med. 2016;374(11):1094.

14. Wiviott SD, Raz I, Bonaca MP, et al. Dapagliflozin and cardiovascular outcomes in type 2 diabetes. N Engl J Med. 2019;380(4):347-57.

15. Hupfeld C, Mudaliar S. Navigating the "MACE" in cardiovascular outcomes trials and decoding the relevance of atherosclerotic cardiovascular disease benefits versus heart failure benefits. Diabetes Obes Metab. 2019;21(8):1780-9.

16. Scheen AJ. Implications of the recent CVOTs in type 2 diabetes: impact on guidelines: the endocrinologist point of view. Diabetes Res Clin Pract. 2019. https://doi.org/10.1016/j.diabres.2019.05. 005.

17. Frieden TR. Evidence for health decision makingbeyond randomized, controlled trials. N Engl J Med. 2017;377(5):465-75.

18. Kosiborod M, Cavender MA, Fu AZ, et al. Lower risk of heart failure and death in patients initiated on sodium-glucose cotransporter- 2 inhibitors versus other glucose-lowering drugs: the CVD-REAL study (comparative effectiveness of cardiovascular outcomes in new users of sodium-glucose cotransporter-2 inhibitors). Circulation. 2017;136(3): 249-59.

19. Toulis KA, Willis BH, Marshall T, et al. All-cause mortality in patients with diabetes under treatment with dapagliflozin: a population-based, open-cohort study in the Health Improvement Network 
database. J Clin Endocrinol Metab. 2017;102(5): 1719-25.

20. Nystrom T, Bodegard J, Nathanson D, et al. Novel oral glucose-lowering drugs are associated with lower risk of all-cause mortality, cardiovascular events and severe hypoglycaemia compared with insulin in patients with type 2 diabetes. Diabetes Obes Metab. 2017;19(6):831-41.

21. Norhammar A, Bodegard J, Nystrom T, et al. Dapagliflozin and cardiovascular mortality and disease outcomes in a population with type 2 diabetes similar to that of the DECLARE-TIMI 58 trial: a nationwide observational study. Diabetes Obes Metab. 2019;21(5):1136-45.

22. Dalan R. Sodium-glucose cotransporter-2 inhibition in type 2 diabetes mellitus: a review of large-scale cardiovascular outcome studies and possible mechanisms of benefit. Cardiol Rev. 2018;26(6): 312-20.
23. Fadini GP, Zatti G, Consoli A, et al. Rationale and design of the DARWIN-T2D (dapagliflozin real world evidence in type 2 diabetes): a multicenter retrospective nationwide Italian study and crowdsourcing opportunity. Nutr Metab Cardiovasc Dis. 2017;27(12):1089-97.

24. Fadini GP, Sciannameo V, Franzetti I, et al. Similar effectiveness of dapagliflozin and GLP-1 receptor agonists concerning combined endpoints in routine clinical practice: a multicentre retrospective study. Diabetes Obes Metab. 2019;21:1886-94.

25. Wittbrodt E, Chamberlain D, Arnold SV, et al. Eligibility of patients with type 2 diabetes for sodiumglucose co-transporter-2 inhibitor cardiovascular outcomes trials: an assessment using the diabetes collaborative registry. Diabetes Obes Metab. 2019;21:1985-9. 